\title{
Auch ältere Patienten profitieren von autologer Stammzelltransplantation
}

\begin{abstract}
Auch bei älteren Patienten mit neu diagnostiziertem multiplem Myelom kann eine autologe Stammzelltransplantation (ASCT) das Überleben verbessern. Dies ergab eine aktuelle Analyse aus dem Deutschen Register für Stammzelltransplantation. Daten von insgesamt 3.591 Patienten im Alter von 60 bis 79 Jahren flossen in die Analyse ein. Verglichen wurde das relative Überleben mit demjenigen von Patienten mit multiplem Myelom, die im gleichen Zeitraum ihre Diagnose erhalten hatten und im bevölkerungsbasierten deutschen Krebsregister registriert worden waren $(n=13.903)$. In der ASCT-Kohorte war das mittlere Alter mit 65 gegegnüber 70 Jahren niedriger.
\end{abstract}

Die Zahl der autologen Stammzelltransplantationen stieg von 109 in den Jahren 1998-2000 auf 1.133 in den Jahren 2010-2012. In allen Altersgruppen zeigte sich beim Vergleich mit der allgemeinen Myelom-Population eine Zunahme des relativen 5-Jahres-Überlebens infolge der ASCT: $60-64$-Jährige: 59,2 vs. $66,1 \%$; 65-69-Jährige: 57,4 vs. $61,7 \%$; 70-79-Jährige: 51,0 vs. $56,6 \%$.

Das relative Überleben verbesserte sich von 2003-2005 gegenüber 2009-2011 in der allgemeinen deutschen Myelom-Population um 8,5\%, in der mit Stammzelltransplantation behandelten Kohorte um $11,8 \%$. Die Unterschiede zwischen den beiden Kohorten im relativen Überleben

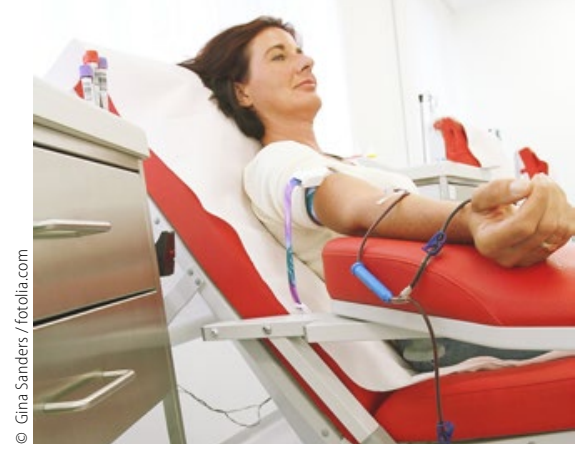

Stammzellen können das Leben von Myelompatienten in jedem Alter verlängern.

stiegen, von 1,9\% auf 5,2\% zugunsten der ASCT-Kohorte.

Wie jüngere Patienten hatten auch ältere Patienten, die eine komplette Remission nach der ASCT erreichten, Überlebensvorteile. Dies, so die Forscher um Maximilian Merz, deute an, dass auch bei älteren Patienten mit multiplem Myelom die Tiefe der Remission ein Behandlungsziel sein müsse.

Kathrin von Kieseritzky

Merz M et al. Survival of elderly patients with multiple myeloma - Effect of upfront autologous stem cell transplantation. Eur J Cancer. 2016;62:1-8.

\section{All-trans-Retinsäure und Arsentrioxid in Kombination}

Die finalen Ergebnisse der APL0406-Studie zeigen, dass die Kombination Alltrans-Retinsäure (ATRA) plus Arsentrioxid (ATO) bei der Ersttherapie der akuten promyelozytischen Leukämie (APL) mit niedrigem bis intermediärem Risiko gegenüber der Standardtherapie mit ATRA plus Chemotherapie (CHT) von Vorteil sein kann. Im ATRA/ATO-Arm erreichten alle 127 Patienten und im ATRA/CHT-Arm 132 von 136 Patienten eine komplette Remission ( $\mathrm{p}=0,12$ ). 259 Patienten durchliefen die Konsolidierungstherapie. Auch nach dem dritten Konsolidierungszyklus wiesen alle Patienten im ATRA/ATO-Arm keine PMLPARA-Translokation auf, während im ATRA/CHT-Arm zwei (1,7\%) Patienten nun positiv getestet wurden, und damit eine molekulare Resistenz aufwiesen. Nach einem medianen Follow-up von 40,6 Monaten betrug die Rate für das er-

\section{kurz notiert}

\footnotetext{
Auf negativen Befund im Brust-MRT ist Verlass

In einer Metaanalyse untersuchten Forscher der Universität Wien die Verlässlichkeit der Magnetresonanztomografie (MRT) bei Brustkrebsverdacht [Bennani-Baiti B et al. PLoS ONE 2016;11(8):e0160346]. Insgesamt wurden 2.316 verdächtige Befunde der Brust, betrachtet, die zuvor in Mammografie oder Ultraschall erhoben worden waren. Eine Mikrokalzifizierung lag nicht vor. Aus den gepoolten Daten errechneten Bennani-Baiti und Mitarbeiter für die MRT bei Brustkrebsverdacht eine Sensitivität von $99 \%$, eine Spezifität von $89 \%$, einen positiven Vorhersagewert von $56 \%$ und einen negativen Vorhersagewert von $100 \%$. Auf einen negativen MRT-Befund ist somit Verlass. Weniger aussagekräftig sind positive Befunde im Brust-MRT. Die relativ niedrige Spezifität führt zu einer großen Zahl falsch positiver Resultate mit entsprechenden Konsequenzen. Der positive Vorhersagewert liegt daher nur bei $56 \%$. Dabei ist aber zu berücksichtigen, dass der Vorhersagewert von positiven Befunden in der Mammografie noch deutlich darunter liegt, nämlich bei $14 \%$. Robert Bublak
}

eignisfreie Überleben 97,3 versus $80 \%$ (p $<0,001)$, die kumulative Rezidivinzidenz 1,9 versus $13,0 \%(\mathrm{p}=0,0013)$ und die Rate für das 50-Monats-Gesamtüberleben 99,2 versus $92,6 \%(p=0,0073)$.

Alle 117 Patienten im ATRA/CHTArm, die die Konsolidierung vollständig durchliefen und PML-RARA-negativ getestet waren, bekamen eine Erhaltungstherapie. Neun dieser Patienten erlebten ein Rezidiv.

Nach der Induktion kam es im ATRA/ ATO-Arm zu zwei Rezidiven und einem Todesfall in kompletter Remission, im ATRA/CHT-Arm zu zwei Fällen molekularer Resistenz nach dem dritten Konsolidierungszyklus, 15 Rezidiven und fünf Todesfällen in kompletter Remission. Zwei Patienten im ATRA/CHT-Arm entwickelten eine therapiebedingte myeloische Neoplasie.

Kathrin von Kieseritzky

Platzbecker U et al. Improved Outcomes With Retinoic Acid and Arsenic Trioxide Compared With Retinoic Acid and Chemotherapy in Non-HighRisk Acute Promyelocytic Leukemia: Final Results of the Randomized Italian-German APL0406 Trial. J Clin Oncol. 2016;Jul 11 (Epub ahead of print). 